\title{
Injury to the Cerebellum in Term Asphyxiated Newborns Treated with Hypothermia
}

\author{
D. Kwan, E. Boudes, G. Gilbert, C. Saint-Martin, S. Albrecht, M. Shevell, and DP. Wintermark
}

\begin{abstract}
BACKGROUND AND PURPOSE: Until now, most studies of brain injury related to term neonatal encephalopathy have focused on the cerebrum and ignored the cerebellum. We sought to evaluate whether cerebellar injury occurs in term asphyxiated neonates.
\end{abstract}

MATERIALS AND METHODS: Asphyxiated neonates treated with hypothermia were enrolled prospectively. Severity of brain injury in the cerebrum was scored on each MR imaging obtained during the first month of life; cerebellar injury was recorded when mentioned in the imaging or autopsy report. In addition, for some of the neonates, the ADC and fractional anisotropy were measured in 4 regions of interest in the cerebellum.

RESULTS: One hundred seventy-two asphyxiated neonates met the criteria for hypothermia. Cerebellar injury was visible only on conventional imaging of $4 \%$ of the neonates for whom brain imaging was available, but it was reported in the autopsy report of $72 \%$ of the neonates who died. In addition, 41 of the asphyxiated neonates had a total of 84 ADC and fractional anisotropy maps. Neonates with brain injury described only in the cerebrum demonstrated ADC and fractional anisotropy changes similar to those of the neonates with brain injury in the cerebrum and cerebellum -increased ADC around day 10 of life and decreased fractional anisotropy on day 2-3 of life, around day 10 of life, and around 1 month of age.

CONCLUSIONS: The cerebellum may be injured in term neonates after birth asphyxia. These cerebellar injuries are only rarely visible on conventional imaging, but advanced neuroimaging techniques may help to identify them.

ABBREVIATION: FA = fractional anisotropy

B irth asphyxia and its resulting neonatal encephalopathy occur in approximately 2 per 1000 births in developed countries and are responsible for $23 \%$ of all neonatal deaths worldwide. ${ }^{1-3}$ Children who survive birth asphyxia may develop severe neurodevelopmental complications, such as cerebral palsy, mental retardation, learning difficulties, and other cognitive deficits. Until now, therapeutic hypothermia to an esophageal temperature of $33.5^{\circ} \mathrm{C}$ initiated within the first 6 hours of life and continued for 72 hours has emerged as the only available safe treatment for possibly im-

Received December 16, 2014; accepted after revision February 2, 2015.

From the Division of Newborn Medicine, Department of Pediatrics (S.K., E.B., P.W.) Division of Pediatric Radiology (C.S.-M.), Department of Pediatric Pathology (S.A.), and Division of Pediatric Neurology, Department of Pediatrics (M.S.), Montreal Children's Hospital, McGill University, Montreal, Quebec, Canada; and MR Clinical Science (G.G.), Philips Healthcare, Montreal, Quebec, Canada.

Please address correspondence to Pia Wintermark, MD, McGill University/Montreal Children's Hospital, Division of Newborn Medicine, 1001 boul Décarie, Site Glen, Block E, EM0.3244, Montréal, QC H4A 3J1, Canada; e-mail: pia.wintermark@bluemail.ch

- Indicates open access to non-subscribers at www.ajnr.org

E Indicates article with supplemental on-line table.

http://dx.doi.org/10.3174/ajnr.A4326 proving the long-term outcome of some of these asphyxiated neonates. $^{4-6}$

Parents of these neonates are wondering increasingly about the long-term prognosis while their neonate is still hospitalized in the neonatal intensive care unit. In the past, the prognosis was based on clinical history, clinical examinations, and neurologic tools such as electroencephalography. ${ }^{7}$ In the last few years, MR imaging has developed considerably, and conventional MR imaging sequences have become the standard for visualizing the presence and extent of brain injury after hypothermia treatment is completed. ${ }^{8,9}$ However, newer advanced MR imaging modalities, such as DTI and subsequent apparent diffusion coefficient and fractional anisotropy (FA) maps, are becoming available tools for use with neonates and may permit an even better definition of brain injury and thus may provide a more accurate prognosis. ${ }^{10,11}$

Typical patterns of injury in term asphyxiated neonates are usually described in the cerebrum, including basal ganglia injury, watershed injury, and near-total injury. Until now, injury to the cerebellum has been reported only rarely after birth asphyxia in term neonates. Nevertheless, the few available imaging studies in 
which the cerebellum was examined reported cerebellar injury in severe cases of neonatal encephalopathy. ${ }^{12-15}$ Sargent et al ${ }^{15}$ found cerebellar vermian atrophy on CT and MR imaging acquired from 12 neonates around 10-19 months of age with neonatal encephalopathy and who developed thalamic injury with or without cortical injury; however, they did not find any signs of cerebellar involvement on the imaging performed on these patients during the first 2 weeks of life. Le Strange et al ${ }^{14}$ confirmed reduced cerebellar growth, including vermis growth, during the first year after birth in 6 neonates with neonatal encephalopathy who developed severe basal ganglia and thalamic injury. Connolly et $\mathrm{al}^{13}$ found abnormally high T2 signal intensity in the anterior lobe of the vermis on MR images acquired at various ages (ie, from 1 to 24 years) from 18 neonates with neonatal encephalopathy who had developed near-total injury. As demonstrated in premature neonates, ${ }^{16}$ studying the cerebellum in neonates is highly relevant and important, because injury to the cerebellum may create an additional burden on the future neurodevelopment of these neonates.

Thus, this present study was designed to report the incidence of cerebellar injury in asphyxiated neonates treated with hypothermia. We hypothesize that asphyxiated neonates are at risk of injury to the cerebellum and that cerebellar injury may be better identified in these neonates by using advanced neuroimaging techniques.

\section{MATERIALS AND METHODS \\ Patients}

We conducted a prospective cohort study of term asphyxiated neonates admitted to our neonatal intensive care unit between 2008 and 2014 and who met the criteria for induced hypothermia $^{4-6}: 1$ ) gestational age of $\geq 36$ weeks and birth weight of $\geq 1800$ $\mathrm{g} ; 2$ ) evidence of fetal distress, such as history of an acute perinatal event, cord $\mathrm{pH}$ of $\leq 7.0$, or base deficit of $-16 \mathrm{mEq} / \mathrm{L}$ or lower; 3 ) evidence of neonatal distress, such as an Apgar score of $\leq 5$ at 10 minutes, postnatal blood gas $\mathrm{pH}$ obtained within the first hour of life of $\leq 7.0$, or a base deficit of $-16 \mathrm{mEq} / \mathrm{L}$ or lower, or continued need for ventilation initiated at birth and continued for at least 10 minutes; and 4) evidence of moderate or severe encephalopathy obtained by physical examination and/or amplitude-integrated electroencephalography. Eligible patients received whole-body cooling to an esophageal temperature of $33.5^{\circ} \mathrm{C}$, initiated by 6 hours of life and continued for 72 hours (unless contraindications developed), and then slow rewarming. The research protocol was approved by the Montreal Children's Hospital institutional review board, and informed parental consent was obtained in each case.

\section{Brain MR Imaging}

Per standard clinical protocol at our institution, a brain MR imaging scan was performed for all these neonates around day 10 of life. The MR imaging scans were performed by using a 3T clinical system (Achieva X; Philips Healthcare, Best, the Netherlands). Each MR imaging study included a 3D T1-weighted gradientecho (TR, 24 ms; TE, 4.6 ms; matrix size, $180 \times 180$; FOV, 180 $\mathrm{mm}$; flip angle, $30^{\circ}$; sagittal sections, 104 ; section thickness, 1.0 $\mathrm{mm}$; and multiplanar reformations in axial and coronal planes), a
TSE high-resolution T2-weighted (TR, $5000 \mathrm{~ms}$; TE, $90 \mathrm{~ms}$; TSE factor, 15; matrix size, $300 \times 300$; FOV, $150 \mathrm{~mm}$; flip angle, $90^{\circ}$; axial sections, 27; section thickness, $3.0 \mathrm{~mm}$ ), and a single-shot EPI DWI (TR, $2424.4 \mathrm{~ms}$; TE, $69 \mathrm{~ms}$; matrix size, $200 \times 117$; FOV, $240 \mathrm{~mm}$; b-values, 600 and $1200 \mathrm{~s} / \mathrm{mm}^{2}$; flip angle, $90^{\circ}$; axial sections, 21 ; section thickness, $4.0 \mathrm{~mm}$ ) sequence. These 3 sequences combined are referred to as "conventional MR imaging" throughout the remainder of this article, because they were the ones used by the neuroradiologists to interpret the MR imaging studies and compile their reports.

Pediatric neuroradiologists, who were blind to the clinical conditions of the infants, interpreted the MR imaging studies of the asphyxiated neonates treated with hypothermia. They reported the presence and extent of brain injury in the cerebrum according to a previously described MR imaging scoring system. ${ }^{17}$ Cerebellar injury was recorded when mentioned in their report to evaluate the incidence of cerebellar injury in these neonates obtained by conventional imaging. The presented data were based only on the reports from the initial time of the study. We wanted to focus on the reading of the conventional sequences by the neuroradiologists to determine how often cerebellar injury was reported. We did not want to influence or bias them by asking them to specifically review the images for the cerebellum.

\section{Autopsy}

If the neonate did not survive, the autopsy result (when available) was also used to assess the presence and extent of brain injury in the cerebrum and the cerebellum. Cerebellar injury was recorded when mentioned in the report to evaluate the incidence of cerebellar injury in these neonates obtained by pathology.

\section{ADC and FA Maps}

In addition, since 2010, when possible (ie, when the parents consented for their neonate to have additional MR imaging, when the neonates were hemodynamically stable, and when a team of a nurse and a respiratory therapist was available to assist with the MR imaging), neonates were enrolled in an MR imaging research study, and MR imaging scans were performed on day 1 of life, day 2-3 of life, around day 10 of life, and/or around 1 month of life. These time points were chosen to ensure the absence of antenatal brain injury (day 1 of life), to assess early patterns of injury (day 2-3 of life), and to define the extent of definitive brain injuries (around day 10 of life and around 1 month of life). Patients who underwent hypothermia had therapy maintained during the MR imaging scan without any adverse events. ${ }^{18}$ Any ventilation, pressor support, or sedation was maintained during the MR imaging process, and additional sedation was avoided.

A similar imaging protocol was used with these neonates at different time points. However, in addition to the above-mentioned sequences, a single-shot EPI DTI sequence with isotropic resolution (TR, $5937.8 \mathrm{~ms}$; TE, $69 \mathrm{~ms}$; matrix size, $100 \times 100$; FOV, $180 \mathrm{~mm}$; sensitivity encoding factor, 2; directions, 32; bvalue, $750 \mathrm{~s} / \mathrm{mm}^{2}$; axial sections, 46 ; section thickness, $1.8 \mathrm{~mm}$ ) was included in the imaging protocol for the neonates enrolled in the MR imaging research study. In addition, the FSL Brain Extraction Tool (http://fsl.fmrib.ox.ac.uk/fsl/fslwiki/BET) was used to remove all extracerebral tissues (ie, eyes, meninges, skull, etc) 
from the images. ${ }^{19}$ Then, ADC and FA maps were generated for the whole brain by using the tools of the Diffusion Imaging in Python (DIPY) package (Sherbrooke Connectivity Imaging Lab, Sherbrooke University, Sherbrooke, Quebec, Canada). ${ }^{20}$

To assess whether subtle changes in the cerebellum were not recognized on conventional MR imaging of these patients, ADC and FA values were measured in 4 different regions of interest in the cerebellum (ie, the superior cerebellar peduncle, the dentate nucleus, the middle cerebellar peduncle, and the inferior cerebellar peduncle), as described in other study reports. ${ }^{21-23}$ The regions of interest were always drawn by the same observer by using ImageJ (Image Processing and Analysis in Java; National Institutes of Health, Bethesda, Maryland). ${ }^{24}$ Axial T2-weighted imaging was used in conjunction with the axial ADC and FA maps to accurately identify the regions of interest in the cerebellum. Measurements in the right and left sides in these different regions of interests were obtained and then averaged. The measurements were repeated twice for each region of interest to increase reproducibility.

\section{Statistical Analysis}

For the analysis of ADC and FA measurements, asphyxiated neonates treated with hypothermia were categorized into 1 of 3 subgroups on the basis of the presence or absence of brain injury reported from MR imaging and/or autopsy: 1) neonates who did not develop injury, 2) neonates who developed brain injury in the cerebrum, and 3) neonates who developed brain injury in the cerebrum and the cerebellum. Statistical analysis was performed according to the day of life on which MR imaging was performed. For each region of interest, ADC and FA values were compared between the groups by using Mann-Whitney $U$ tests. A $P$ value of $<.05$ was considered statistically significant. All statistical analyses were performed by using GraphPad Prism software (GraphPad Software, San Diego, California).

\section{RESULTS}

\section{Description of the Patients}

A total of 172 term asphyxiated neonates met the criteria for hypothermia (On-line Table).

Of the asphyxiated neonates, 83\% (143 of 172) survived. Among these patients, only $3 \%$ ( 5 of 143 ) had a cerebellar injury described on their MR imaging report; these patients presented with an associated watershed injury or with a near-total injury. Injury to the cerebellum was described most often as an abnormal T1 signal in specific regions of the cerebellum (the dentate nuclei or surrounding cerebellar white matter) and/or, more rarely, as a restricted diffusion in the cerebellum. Among the remaining patients without a described cerebellar injury, 56\% (77 of 138) did not develop brain injury in the cerebrum, and $44 \%$ (61 of 138) did develop brain injury in the cerebrum; 20\% (12 of 61) developed basal ganglia injury, 44\% (27 of 61) developed watershed injury, and $36 \%$ (22 of 61) developed near-total injury. It is notable that 2 neonates had small hematomas within the left cerebellar hemisphere, and 1 neonate had one within the right cerebellar hemisphere; several neonates presented with subdural hematomas in the posterior cerebellar hemisphere, but these hematomas did not exert any mass effect on the underlying cerebellum.
Seventeen percent (29 of 172) of the neonates died as a result of complications of birth asphyxia and neonatal encephalopathy. Among those who died, 48\% (14 of 29) had an available autopsy report that described brain injury; 71\% (10 of 14) presented with apoptosis clearly identified in the cerebellum at the time of autopsy, 21\% (3 of 14) presented with acidophilic neurons in the cerebellum, and 7\% (1 of 14) did not have any described injury in the cerebellum. Each patient who died and had an autopsy had brain injury in the cerebrum at the time of the autopsy. It is interesting to note that among the neonates who died and had an autopsy, 2 had brain MR imaging before death, but the presence of cerebellar injury was mentioned in only one of the neonate's MR imaging reports. With respect to this patient born after placental abruption (Fig 1), a restricted diffusion and an increased T1 signal were reported around the bilateral dentate nuclei of the cerebellum; the autopsy of this patient revealed numerous apoptotic neurons throughout the internal granular layer of the cerebellar cortex and a necrosis of the dentate nuclei and the Purkinje cells in the cerebellar cortex. Among the 52\% (15 of 29) of neonates who died and did not have an autopsy, only $27 \%$ (4 of 15) had brain MR imaging performed, and the presence of cerebellar injury was recognized in only one of the MR imaging reports. In this patient, restricted diffusion was again noted on the ADC map around the bilateral dentate nuclei.

Among the 16 asphyxiated neonates with cerebellar injury reported from their MR imaging and/or autopsy, 3 were born after placental abruption, 1 was born after cord prolapse, 1 was born after placenta previa, 2 were born with tight nuchal cord, 6 presented profound fetal bradycardia of unclear origin, 1 presented poor variability, and 2 did not have a clear cause to explain their asphyxial event.

\section{ADC and FA Maps}

A total of $84 \mathrm{ADC}$ and FA maps were obtained for 41 asphyxiated neonates treated with hypothermia, in whom a DTI sequence was performed in addition to the conventional sequences. Ten were obtained on day 1 of life, 22 on day 2-3 of life, 32 around day 10 of life (mean, day 10 of life; range, day $7-15$ of life), and 20 around 1 month of life (mean, day 33 of life; range, day 29-42 of life).

ADC values (Fig 2) were significantly increased around day 10 of life in the superior cerebellar peduncle $(P=.0001)$, the middle cerebellar peduncle $(P=.0004)$, and the inferior cerebellar peduncle $(P=.006)$ in neonates with brain injury of the cerebrum and cerebellum compared with those without brain injury; the $\mathrm{ADC}$ values in the dentate nucleus were not different around day 10 of life between these 2 groups of neonates. In addition, ADC values were not different between these 2 groups at the other time points except on day 1 of life for the middle cerebellar peduncle $(P=.003)$, for which the ADC was significantly increased in the neonates with brain injury in the cerebrum and cerebellum. ADC values in the cerebellum were not different between neonates with a brain injury described only in the cerebrum and those without injury except around day 10 of life for the superior cerebellar peduncle $(P<.0001)$, for which the ADC was significantly increased in the neonates with a brain injury described only in the cerebrum.

FA values (Fig 3) were significantly decreased around day 10 of 


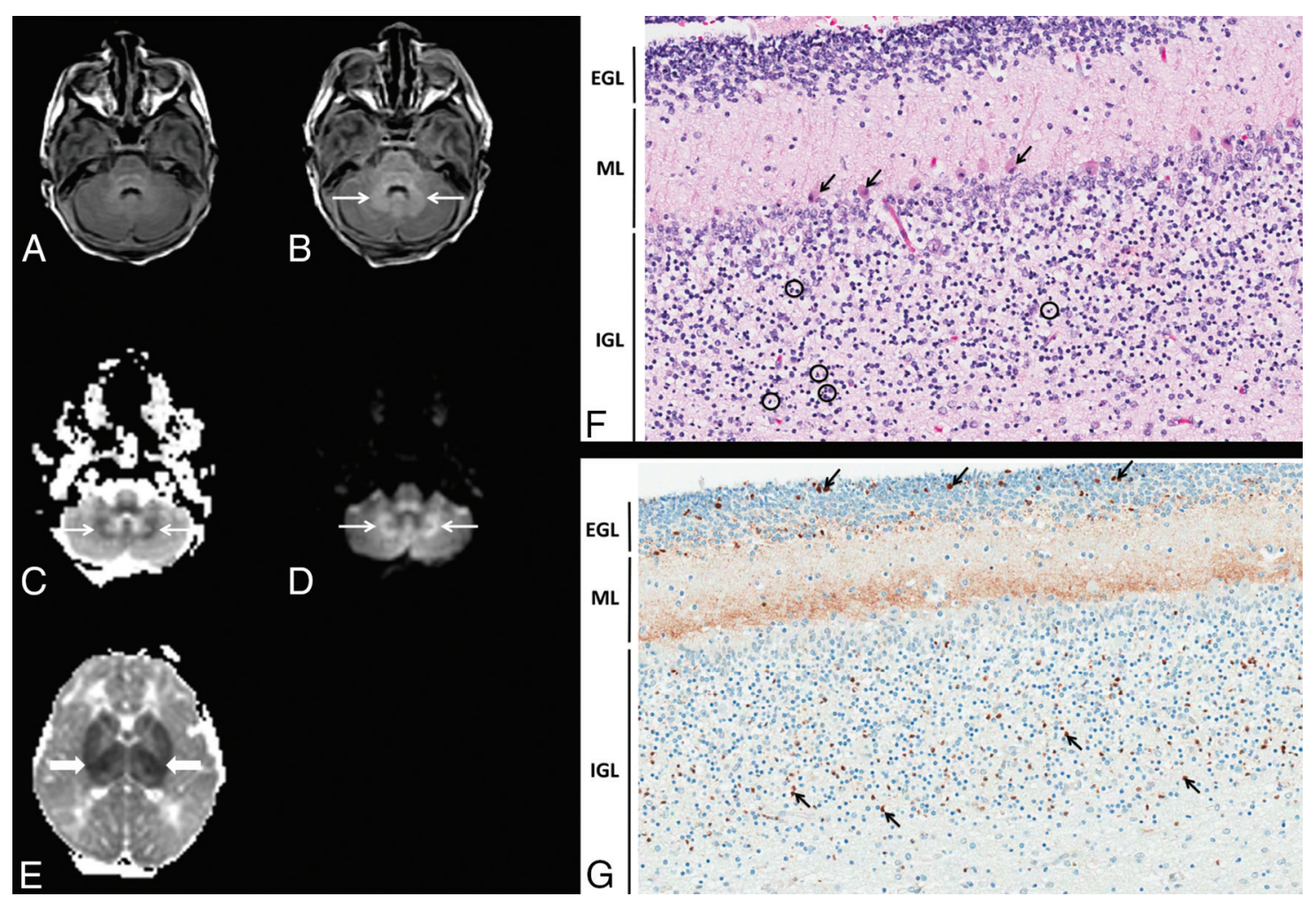

FIG 1. Term asphyxiated neonate treated with hypothermia who presented with cerebellar injury on MR imaging and histopathology. A, The cerebellum on day 1 of life (axial T1-weighted image shows a normal signal in the cerebellum). $B-D$, The cerebellum on day 2 of life. Axial T1-weighted image $(B), A D C$ map $(C)$, and diffusion-weighted image $(D)$ show an abnormal signal (thin arrows) around the bilateral dentate nuclei. $E$, ADC map of the cerebrum on day 2 of life shows an associated marked basal ganglia injury (thick arrows). $F$ and G, Histopathology of the cerebellum on day 3 of life (magnification $\times 20$ ). Hematoxylin-eosin-stained section of the cerebellar cortex $(F)$ shows the Purkinje cells (arrows) with shrunken hypereosinophilic cytoplasm and condensed nuclei, typical of hypoxic-ischemic necrosis ("red and dead" neurons). In addition, in the internal granular layer (IGL), many apoptotic internal granular neurons (circles) are present. G, Immunostaining of the cerebellar cortex for active caspase 3 (orange-brown staining), highlights the apoptotic internal granular neurons. In addition, the staining of the neuropil of the molecular layer (ML) is consistent with "synapoptosis." Also, apoptotic neuronal precursor cells (arrows) are present in the external granular layer (EGL), which occurs as part of the normal involution of this layer.

life in the superior cerebellar peduncle $(P=.007)$, the middle cerebellar peduncle $(P<.0001)$, and the inferior cerebellar peduncle $(P<.0001)$ in neonates with brain injury of the cerebrum and cerebellum compared with those without brain injury; the FA values in the dentate nucleus were not different around day 10 of life between these 2 groups of neonates. Similarly, a decreased FA value also was observed in the 1 neonate with a brain injury of the cerebrum and cerebellum, which was scanned around 1 month of age. The same trend was already observed on day $2-3$ of life in the middle cerebellar peduncle $(P<.0001)$ and the inferior cerebellar peduncle $(P=.0004)$ in the neonates with brain injury of the cerebrum and cerebellum compared with those without brain injury. It is interesting to note that the FA values significantly decreased around 1 month of life in the superior cerebellar peduncle $(P<.0001)$, the middle cerebellar peduncle $(P<.0001)$, and the inferior cerebellar peduncle $(P<.0001)$ in the neonates with a brain injury described only in the cerebrum compared with those without brain injury; the FA values in the dentate nucleus were not different around 1 month of life between these 2 groups of neonates. The same trend was already observed on day 2-3 of life and around day 10 of life in the middle cerebellar peduncle $(P<.0001$ on day $2-3$ of life and $P=.03$ around day 10 of life) and in the inferior cerebellar peduncle $(P=.07$ on day $2-3$ of life and $P=.003$ around day 10 of life) in the neonates with brain injury of the cerebrum and cerebellum compared with those without brain injury.

\section{DISCUSSION}

The results of this study provide insight into cerebellum injury in asphyxiated neonates treated with hypothermia. Cerebellar injury was only rarely reported on conventional MR imaging. Only 6 asphyxiated neonates treated with hypothermia had a cerebellar injury described on their MR imaging report, which represents $4 \%$ of the asphyxiated neonates treated with hypothermia from the cohort with brain imaging data available, or $9 \%$ of the asphyxiated neonates treated with hypothermia who developed brain injury visible on brain imaging. In these 6 cases, the cerebellar injury was also always associated with an injury of the cerebrum. However, cerebellar injury was a common finding in the autopsies of the asphyxiated neonates treated with hypothermia who died (ie, it was clearly present in $72 \%$ of these neonates). The cerebellar injury was always associated with an injury of the cerebrum. Thus, the incidence of a neuroimaging-evident cerebellar injury in asphyxiated neonates treated with hypothermia widely contrasts with the incidence of pathologically evident cerebellar injury in these neonates, as already pointed out by others. ${ }^{15}$ It is possible that the asphyxiated neonates who died were among the ones with 

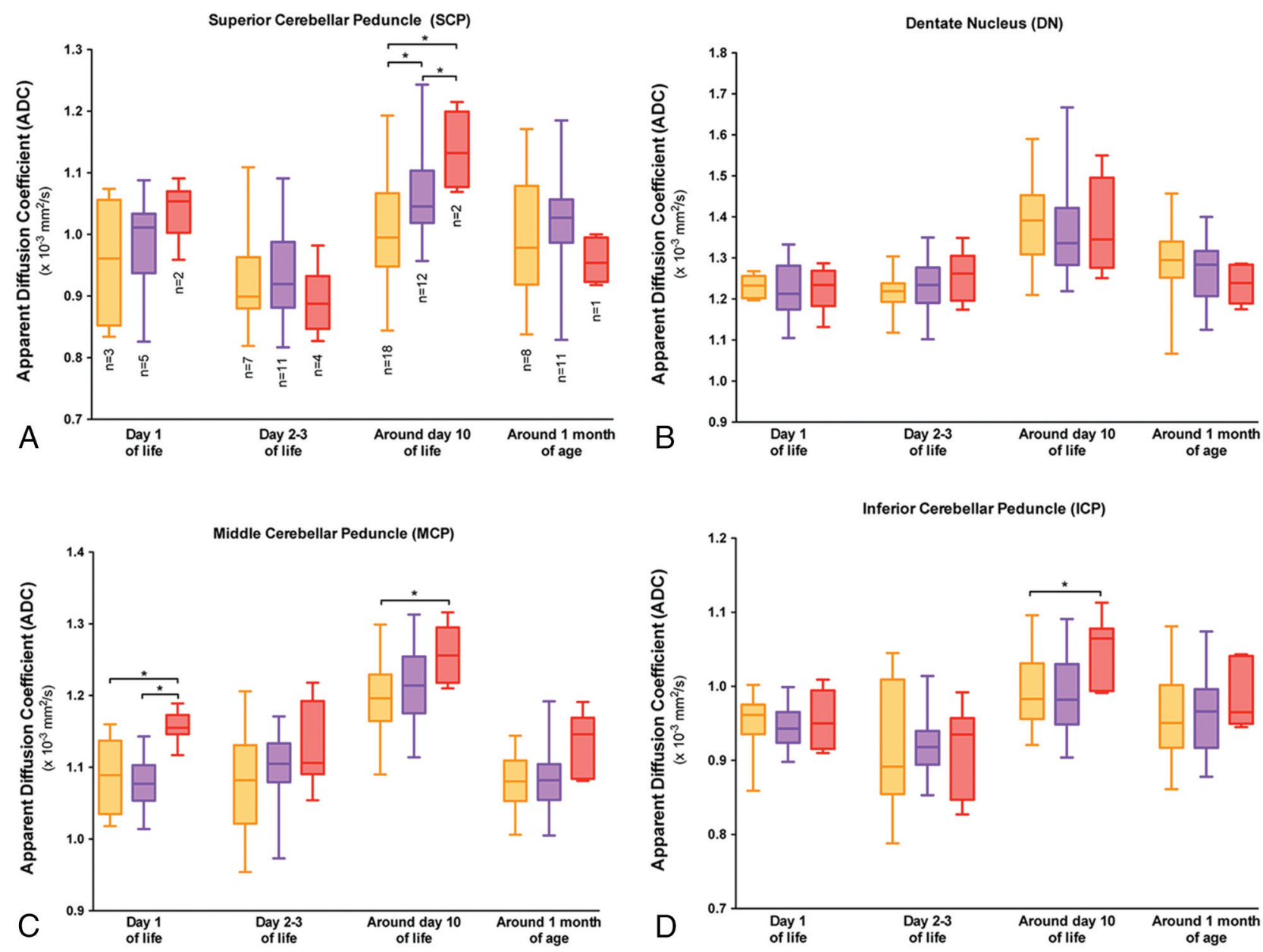

\section{Asphyxiated newborns not developing brain injury}

Asphyxiated newborns developing injury in cerebrum

${ }^{*} p<0.05$

\section{Asphyxiated newborns developing injury in cerebrum and cerebellum}

FIG 2. Comparison of ADC values in each region of interest in the cerebellum among the neonates who did not develop injury, neonates who developed brain injury in the cerebrum, and neonates who developed brain injury in the cerebrum and the cerebellum on day 1 of life, day 2-3 of life, around day 10 of life, and around day 30 of life. Each box and pair of whiskers indicate the median and the minimum and maximum ADC values, respectively. The different regions of interest are the superior cerebellar peduncle $(A)$, the dentate nucleus $(B)$, the middle cerebellar peduncle $(C)$, and the inferior cerebellar peduncle $(D)$.

the most severe brain injury. It is also possible that the neuroradiologists interpreting the conventional imaging concentrated their attention on the supratentorial injury and overlooked the cerebellar injury when reporting their results. However, it is also possible that cerebellar injuries are subtle on conventional imaging and hard to recognize ${ }^{14,15}$ and that they are only clearly visible in asphyxiated neonates with severe brain injury. ${ }^{13}$

In the second part of this study, we used advanced neuroimaging techniques to measure $\mathrm{ADC}$ and $\mathrm{FA}$ in 4 specific regions of the cerebellum. Of the 149 asphyxiated neonates treated with hypothermia in our cohort, 41 (28\% of the neonates with imaging available) had ADC and FA maps available for measurements. Neonates with brain injury of the cerebrum and cerebellum showed ADC and FA changes in some of the measured cerebellar structures, mainly an increased ADC around day 10 of life and a decreased FA on day 2-3 of life and around day 10 of life. It is interesting to note that neonates with brain injury described only in the cerebrum also demonstrated $\mathrm{ADC}$ and FA changes in the same cerebellar structures, again mainly an increased ADC around day 10 of life and a decreased FA on day 2-3 of life, around day 10 of life, and around 1 month of age. Neonates with brain injury described only in the cerebrum (and not in the cerebellum) presented ADC and FA values in their cerebellum that were similar to those in the neonates with brain injury described in the cerebrum and cerebellum. These results suggest an unrecognized cerebellum injury in some of the neonates with brain injury described only in the cerebrum when using conventional MR imaging sequences. It is interesting to note that after this analysis, we went back and examined the conventional imaging of some of these neonates with abnormal FA values, and we found the changes to be very subtle and hard to recognize on conventional imaging. Advanced neuroimaging techniques with measurements of ADC and FA values seem to be able to identify these cerebellum injuries that are not visible on conventional imaging, and thus, 

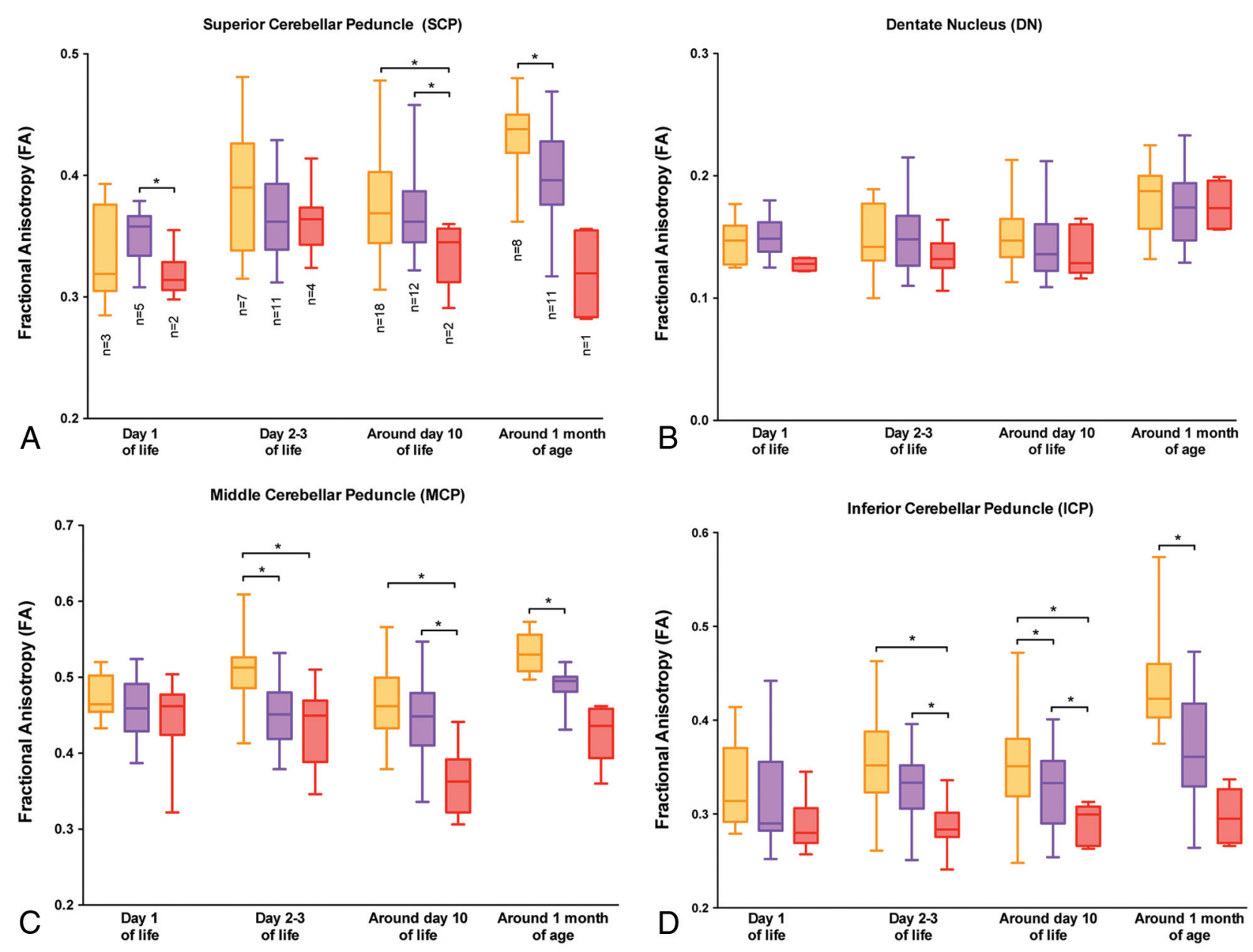

$\square$ Asphyxiated newborns not developing brain injury

$\square$ Asphyxiated newborns developing injury in cerebrum $\square$ Asphyxiated newborns developing injury in cerebrum and cerebellum

FIG 3. Comparison of FA values in each region of interest in the cerebellum between neonates who did not develop injury, neonates who developed brain injury in the cerebrum, and neonates who developed brain injury in the cerebrum and the cerebellum on day 1 of life, day $2-3$ of life, around day 10 of life, and around day 30 of life. Each box and pair of whiskers indicate the median and the minimum and maximum FA values, respectively. The different regions of interest are the superior cerebellar peduncle $(A)$, the dentate nucleus $(B)$, the middle cerebellar peduncle $(C)$, and the inferior cerebellar peduncle $(D)$.

they should be used more widely with these neonates to understand the full extent of their injuries.

Typically, brain injury to the cerebrum after neonatal encephalopathy in term neonates has resulted in reduced ADC values during the first days of life, and pseudonormalization after the first week of life ${ }^{25-27}$; similar but slower changes have been shown in conjunction with hypothermia. ${ }^{10}$ In our study, the changes in the cerebellum did not follow this typical pattern. No consistent acute change in ADC values was observed during the first days of life $\mathrm{e}^{12,15}$; the only consistent changes in ADC values were the isolated increased ADC around day 10 of life. Previously, increased $\mathrm{ADC}$ values have been linked to extracellular edema, whereas decreased ADC values have been linked to cytotoxic edema, among other causes. ${ }^{28}$ Thus, these findings raise a question about whether different mechanisms of injury may be involved in cerebellar injury after neonatal encephalopathy. Fractional anisotropy values tended to decrease around day $2-3$ of life in the cerebellar peduncles, and the decrease became more prominent over time and reached significance around day 30 of life. In brain injury to the cerebrum after neonatal encephalopathy, these changes in FA values have been linked with irreversible ischemia, cell death, and loss of structural integrity. ${ }^{29,30}$ In addition, in our study, no patient was found with an isolated injury in the cerebellum. All the neonates with a cerebellar injury described on their MR imaging or autopsy report also had an associated injury to the cerebrum (ie, in the basal ganglia and/or in the cortex and subcortical white matter). ADC and FA changes in the cerebellum were noted in neonates with an injury to the cerebrum and cerebellum and the neonates with an injury described only in the cerebrum compared with those in the group of neonates without injury. It is interesting to note that the 3 cerebellar peduncles showed similar trends in ADC and FA changes, but the dentate nucleus did not show similar changes.

These results suggest that injury to the cerebellum in term 
asphyxiated neonates treated with hypothermia may directly follow the asphyxial event, but it may be also that injury often occurs indirectly by way of the transneuronal degeneration related to damages in other connected areas of the brain rather than directly by way of damage to the cerebellum at the time of asphyxia. ${ }^{14,15,29,31}$ Transneuronal degeneration has been described as the anterograde and retrograde degeneration of neurons synapsing with neurons injured in the initial lesion, related to a disruption of electrical inputs and outputs. ${ }^{29,32-34}$ This process can lead to the disappearance of entire white matter tracts. ${ }^{32-34}$ The 3 cerebellar peduncles manage the fibers that connect the basal ganglia and widespread cortical areas to the cerebellum. ${ }^{35,36}$ An injury to one of these above-mentioned structures can lead to transneuronal degeneration and programmed cell death through the cerebellar peduncles by way of disruption of these pathways. This hypothesis would help to explain the previously reported reduced cerebellar growth in infants with neonatal encephalopathy ${ }^{14}$ and would support a mechanism of diffuse cerebellar injury rather than a focal injury. ${ }^{12}$

The cerebellum is increasingly being recognized as an important component of the brain with a role in motor functions, ${ }^{37}$ such as coordination and balance, but also with a role in vision, cognition, planning, learning, and language. ${ }^{38,39}$ Cerebellar development is known to continue after birth, with continuing cell differentiation and migration and linear growth during the first year after term birth. ${ }^{14}$ In premature neonates, cerebellar injury has been related to a constellation of long-term neurodevelopmental deficits, including impaired motor sequencing, fine motor incoordination, cognitive dysfunction, autism, and other neuropsychiatric sequelae. ${ }^{38,40}$ Impairment of cerebellar growth has been linked with impairment of brain development. ${ }^{38}$ Thus, injury to the cerebellum in term asphyxiated neonates treated with hypothermia may also have a profound impact on the future neurodevelopment of these infants. These cerebellar injuries may explain why some of the asphyxiated neonates still develop more complex impairments, such as an impairment of motor coordination, memory, learning, or visuospatial processing. ${ }^{4}$ Follow-up studies are needed to determine what the contribution of these cerebellar injuries is to the neurodevelopmental sequelae described later in these patients.

As for other conditions and disorders that affect the cerebellum, ${ }^{21}$ advanced neuroimaging (such as measurements of the apparent diffusion coefficient and fractional anisotropy) has proved to be useful for assessing cerebellar injury in term asphyxiated neonates treated with hypothermia. To our knowledge, our study is the first to show that changes in ADC and FA values can be used to evaluate cerebellar injury in asphyxiated neonates treated with hypothermia. It is unfortunate that ADC and FA maps were not measurable for the whole cohort of patients, because the MR imaging sequences that permitted us to obtain these maps are not yet part of the standard of care for these patients at our institution. It also would have been ideal to study a group of asphyxiated neonates not treated with hypothermia to determine if the hypothermia treatment influenced the magnitude of the severity of the observed cerebellar injuries. However, because cooling is now the standard of care, it is no longer possible to randomly assign infants to undergo or not undergo cooling to assess the impact of hypothermia on cerebellar injuries. Additional studies need to determine whether a wider generalization of the use of such sequences may permit the detection of injuries that are for now invisible on conventional neuroimaging and may improve the prognosis given to the parents of such infants. Neurodevelopmental studies of these patients also are recommended to gain a better understanding of how these injuries unfold into neurodevelopmental impairments. We chose to assess only 4 specific regions in the cerebellum that were easily recognizable from one patient to another to make sure that our measurements were very specific. The method we used did not enable us to measure the separate eigenvectors of the diffusion tensor. Additional studies that measure the 3 principal eigenvectors that can describe diffusion in and around a lesion may provide additional insight into injury evolution and the response of the brain to such an insult. ${ }^{30}$ Moreover, values in healthy controls could rule out the possibility that injury occurs only in the cerebellum in asphyxiated neonates treated with hypothermia. An atlas-based DTI analysis in which these results are compared with those of healthy controls would also enable us to better understand these cerebellar injuries.

To calculate the pathologic incidence of cerebellar injury in these neonates, only a description of apoptosis was considered to indicate cerebellar brain injury, because neuronal apoptosis typically requires $24-48$ hours to develop after a hypoxic-ischemic insult. ${ }^{40}$ Acidophilic changes were not accredited to cerebral brain injury in this study, because they usually take approximately 4-6 hours to develop after a hypoxic-ischemic insult ${ }^{41}$ and thus could represent the dying process.

\section{CONCLUSIONS}

The cerebellum may be injured in term neonates after birth asphyxia. These cerebellar injuries are evident on conventional imaging for only a few neonates and are not visible for most other neonates whose potential cerebellar injuries may be identified only by advanced neuroimaging techniques. A combination of $\mathrm{ADC}$ and FA values may be a useful and reliable tool to use for term asphyxiated neonates treated with hypothermia to quantify and ascertain the extent of their cerebellar injury and its temporal evolution after neonatal encephalopathy. Follow-up studies are needed to determine if these cerebellar injuries predict some of the fine motor and other neurodevelopmental abnormalities that can develop later in these neonates.

\section{ACKNOWLEDGMENTS}

We thank the families and their neonates for participating in this study. Special thanks also are due to the neonatal intensive care unit nurses, neonatal intensive care unit respiratory therapists, and MR imaging technicians who made this study possible. We thank Mr. Octavian Dobrescu for his help with the review of the literature and Mr. Wayne Ross Egers for his professional English correction of the manuscript.

Disclosures: Guillaume Gilbert-UNRELATED: Employment: Philips Healthcare, Comments: Clinical scientist who participates in and supports clinical research performed with academic partners. Michael Shevell—UNRELATED: Expert Testimony: Varied law firms and Canadian Medical Protective Association, Comments: Acts as an expert for both defendants and plaintiffs, primarily in perinatal asphyxia cases with regard to issues pertaining to causation, disability, prognosis, and life expectancy; Grants/Grants Pending: NeuroDevNet Network of Centres of Excellence, ${ }^{*}$ Com- 
ments: Funding for national Canadian Cerebral Palsy Registry. Pia WintermarkRELATED: Grant: research grant funding from the Fonds de recherche du QuébecSanté Clinical Research Scholar Career Award Junior 1, the New Investigator Research Grant from the SickKids Foundation, and the Canadian Institutes of Health Research Institute of Human Development, Child and Youth Health.* *Money paid to the institution.

\section{REFERENCES}

1. Bryce J, Boschi-Pinto C, Shibuya K, et al. WHO estimates of the causes of death in children. Lancet 2005;365:1147-52

2. Kurinczuk JJ, White-Koning M, Badawi N. Epidemiology of neonatal encephalopathy and hypoxic-ischaemic encephalopathy. Early Hum Dev 2010;86:329-38

3. Lawn J, Shibuya K, Stein C. No cry at birth: global estimates of intrapartum stillbirths and intrapartum-related neonatal deaths. Bull World Health Organ 2005;83:409-17

4. Azzopardi D, Strohm B, Marlow N, et al; TOBY Study Group. Effects of hypothermia for perinatal asphyxia on childhood outcomes. N Engl J Med 2014;371:140-49

5. Jacobs SE, Berg M, Hunt R, et al. Cooling for newborns with hypoxic ischaemic encephalopathy. Cochrane Database Syst Rev 2013;1:CD003311

6. Shankaran S, Pappas A, McDonald SA, et al. Childhood outcomes after hypothermia for neonatal encephalopathy. $N$ Engl J Med 2012;366:2085-92

7. Thoresen M, Hellström-Westas L, Liu X, et al. Effect of hypothermia on amplitude-integrated electroencephalogram in infants with asphyxia. Pediatrics 2010;126:e131-39

8. Inder TE, Hunt RW, Morley CJ, et al. Randomized trial of systemic hypothermia selectively protects the cortex on MRI in term hypoxic-ischemic encephalopathy. J Pediatr 2004;145:835-37

9. Rutherford M, Ramenghi LA, Edwards AD, et al. Assessment of brain tissue injury after moderate hypothermia in neonates with hypoxic-ischaemic encephalopathy: a nested substudy of a randomised controlled trial. Lancet Neurol 2010;9:39-45

10. Bednarek N, Mathur A, Inder T, et al. Impact of therapeutic hypothermia on MRI diffusion changes in neonatal encephalopathy. Neurology 2012;78:1420-27

11. Gano D, Chau V, Poskitt KJ, et al. Evolution of pattern of injury and quantitative MRI on days 1 and 3 in term newborns with hypoxicischemic encephalopathy. Pediatr Res 2013;74:82-87

12. Castillo M. Selective vulnerability and the cerebellum in neonates. AJNR Am J Neuroradiol 2007;28:20-21

13. Connolly DJ, Widjaja E, Griffiths PD. Involvement of the anterior lobe of the cerebellar vermis in perinatal profound hypoxia. $A J N R$ Am J Neuroradiol 2007;28:16-19

14. Le Strange E, Saeed N, Cowan FM, et al. MR imaging quantification of cerebellar growth following hypoxic-ischemic injury to the neonatal brain. AJNR Am J Neuroradiol 2004;25:463-68

15. Sargent MA, Poskitt KJ, Roland EH, et al. Cerebellar vermian atrophy after neonatal hypoxic-ischemic encephalopathy. AJNR Am J Neuroradiol 2004;25:1008-15

16. Limperopoulos C, Bassan H, Gauvreau K, et al. Does cerebellar injury in premature infants contribute to the high prevalence of longterm cognitive, learning, and behavioral disability in survivors? $\mathrm{Pe}$ diatrics 2007;120:584-93

17. Barkovich AJ, Hajnal BL, Vigneron D, et al. Prediction of neuromotor outcome in perinatal asphyxia: evaluation of MR scoring systems. AJNR Am J Neuroradiol 1998;19:143-49

18. Wintermark P, Labrecque M, Warfield SK, et al. Can induced hypothermia be assured during brain MRI in neonates with hypoxicischemic encephalopathy? Pediatr Radiol 2010;40:1950-54

19. Smith SM. Fast robust automated brain extraction. Hum Brain Mapp 2002;17:143-55
20. Garyfallidis E, Brett M, Amirbekian B, et al. DIPY, a library for the analysis of diffusion MRI data. Front Neuroinform 2014;8:8

21. Chokshi FH, Poretti A, Meoded A, et al. Normal and abnormal development of the cerebellum and brainstem as depicted by diffusion tensor imaging. Semin Ultrasound CT MR 2011;32:539-54

22. Nicoletti G, Manners D, Novellino F, et al. Diffusion tensor MRI changes in cerebellar structures of patients with familial essential tremor. Neurology 2010;74:988-94

23. Widjaja E, Kis A, Go C, et al. Abnormal white matter on diffusion tensor imaging in children with new-onset seizures. Epilepsy Res 2013;104:105-11

24. Rasband WS. ImageJ. US National Institutes of Health, Bethesda, Maryland; 1997-2004. Available at: http://imagej.nih.gov/ij/. Accessed December 16, 2014

25. Barkovich AJ. MR imaging of the neonatal brain. Neuroimaging Clin N Am 2006;16:117-35, viii-ix

26. McKinstry RC, Miller JH, Snyder AZ, et al. A prospective, longitudinal diffusion tensor imaging study of brain injury in newborns. Neurology 2002;59:824-33

27. Rutherford M, Counsell S, Allsop J, et al. Diffusion-weighted magnetic resonance imaging in term perinatal brain injury: a comparison with site of lesion and time from birth. Pediatrics 2004;114: $1004-14$

28. Ito J, Marmarou A, Barzó P, et al. Characterization of edema by diffusion-weighted imaging in experimental traumatic brain injury. J Neurosurg 1996;84:97-103

29. Groenendaal F, Benders MJ, de Vries LS. Pre-Wallerian degeneration in the neonatal brain following perinatal cerebral hypoxiaischemia demonstrated with MRI. Semin Perinatol 2006;30:146-50

30. Ward P, Counsell S, Allsop J, et al. Reduced fractional anisotropy on diffusion tensor magnetic resonance imaging after hypoxic-ischemic encephalopathy. Pediatrics 2006;117:e619-30

31. Limperopoulos C, Soul JS, Haidar H, et al. Impaired trophic interactions between the cerebellum and the cerebrum among preterm infants. Pediatrics 2005;116:844-50

32. Bouza H, Dubowitz LM, Rutherford M, et al. Prediction of outcome in children with congenital hemiplegia: a magnetic resonance imaging study. Neuropediatrics 1994;25:60-66

33. Mazumdar A, Mukherjee P, Miller JH, et al. Diffusion-weighted imaging of acute corticospinal tract injury preceding Wallerian degeneration in the maturing human brain. AJNR Am J Neuroradiol 2003;24:1057-66

34. De Vries LS, Van der Grond J, Van Haastert IC, et al. Prediction of outcome in new-born infants with arterial ischaemic stroke using diffusion-weighted magnetic resonance imaging. Neuropediatrics 2005;36:12-20

35. Bostan AC, Strick PL. The cerebellum and basal ganglia are interconnected. Neuropsychol Rev 2010;20:261-70

36. Salamon N, Sicotte N, Drain A, et al. White matter fiber tractography and color mapping of the normal human cerebellum with diffusion tensor imaging. J Neuroradiol 2007;34:115-28

37. Allin M, Matsumoto H, Santhouse AM, et al. Cognitive and motor function and the size of the cerebellum in adolescents born very pre-term. Brain 2001;124:60-66

38. Limperopoulos C, Chilingaryan G, Sullivan N, et al. Injury to the premature cerebellum: outcome is related to remote cortical development. Cereb Cortex 2014;24:728-36

39. Van Braeckel KN, Taylor HG. Visuospatial and visuomotor deficits in preterm children: the involvement of cerebellar dysfunctioning. Dev Med Child Neurol 2013;55(suppl 4):19-22

40. Biran V, Verney C, Ferriero DM. Perinatal cerebellar injury in human and animal models. Neurol Res Int 2012;2012:858929

41. Becher JC, Bell JE, Keeling JW, et al. The Scottish perinatal neuropathology study: clinicopathological correlation in early neonatal deaths. Arch Dis Child Fetal Neonatal Ed 2004;89:F399-407 